\title{
CP asymmetries in $D$ decays to two pseudoscalars
}

\author{
Ulrich Nierste* \\ Institute for Theoretical Particle Physics (TTP) \\ Karlsruhe Institute of Technology (KIT) \\ 76131 Karlsruhe, Germany \\ E-mail: ulrich.nierste@kit.edu

\section{Stefan Schacht} \\ Dipartimento di Fisica \\ Università di Torino \& INFN \\ Sezione di Torino, 10125 Torino, Italy \\ E-mail: stefan.schachteto.infn.it
}

\begin{abstract}
This talk addresses two topics related to CP asymmetries in $D$ meson decays to two pseudoscalar mesons. First I discuss how new physics can be distinguished from the Standard Model through two sum rules relating three direct $\mathrm{CP}$ asymmetries each, using the sum rule correlating $D^{0} \rightarrow$ $K^{+} K^{-}, D^{0} \rightarrow \pi^{+} \pi^{-}$, and $D^{0} \rightarrow \pi^{0} \pi^{0}$ for illustration. The other sum rule involves $a_{C P}^{\mathrm{dir}}\left(D^{+} \rightarrow\right.$ $\left.\bar{K}^{0} K^{+}\right), a_{C P}^{\mathrm{dir}}\left(D_{s}^{+} \rightarrow K^{0} \pi^{+}\right)$, and $a_{C P}^{\mathrm{dir}}\left(D_{s}^{+} \rightarrow K^{+} \pi^{0}\right)$. The second topic is the direct CP asymmetry in the decay $D^{0} \rightarrow K_{S} K_{S}$, which is expected to be large in the Standard Model for two reasons: Flavor-SU(3) symmetry suppresses the tree amplitude which enhances the crucial "penguin-totree" ratio and the "penguin" amplitude is dominated by the tree-level $W$ boson exchange between $c$ and $u$ quarks. We find that $\left|a_{C P}^{\mathrm{dir}}\left(D^{0} \rightarrow K_{S} K_{S}\right)\right|$ can be as large as $1.1 \%$ in the Standard Model. We advocate $D^{0} \rightarrow K_{S} K_{S}$ as a discovery channel for charm CP violation.
\end{abstract}

38th International Conference on High Energy Physics 3-10 August 2016

Chicago, USA

\footnotetext{
*Speaker.
} 


\section{Introduction}

$\mathrm{CP}$ asymmetries in the charm system play a special role in the search for new physics, because they probe flavor-changing transitions among up-type quarks. At present, a prime effort of experimental charm physics is the discovery of CP violation in charm decays. Within the Standard Model (SM) charm CP asymmetries are small, because the relevant combination of elements of the Cabibbo-Kobayashi-Maskawa (CKM) matrix is of order $10^{-3}$. Precise theoretical predictions are very difficult and vary by several orders of magnitude [1-15]. Since experimental sensitivities have increased to a level that measurements probe the range of SM predictions, we need new ideas to draw the demarcation line between SM and new physics more precisely. In this talk I discuss twobody weak decays of $D^{+}, D^{0}, D_{s}^{+}$mesons into two pseudoscalar mesons $P, P^{\prime}=\pi^{0}, \pi^{ \pm}, K_{S, L}, K^{ \pm}$. My two topics are

(i) sum rules relating $\mathrm{CP}$ asymmetries in three different $D$ decays and

(ii) the $\mathrm{CP}$ asymmetry in the decay $D^{0} \rightarrow K_{S} K_{S}$.

Topic (i) addresses a test of the Standard Model which will only work, if at least one of the involved CP asymmetries is measured non-zero. On the contrary, topic (ii) is about a discovery mode for charm CP violation for the case that the Kobayashi-Maskawa phase is the only source of CP violation.

Charm decay amplitudes are classified in terms of powers of the Wolfenstein parameter

$$
\lambda \simeq\left|V_{u s}\right| \simeq\left|V_{c d}\right| \simeq 0.22
$$

Amplitudes with $A \propto\left\{\begin{array}{l}\lambda^{0} \\ \lambda^{1} \\ \lambda^{2}\end{array}\right\}$ are called $\left\{\begin{array}{l}\text { Cabibbo-favoured (CF) } \\ \text { singly Cabibbo-suppressed (SCS) } \\ \text { doubly Cabibbo-suppressed (DCS) }\end{array}\right\}$. In SCS amplitudes three CKM structures appear,

$$
\lambda_{d}=V_{c d}^{*} V_{u d}, \quad \lambda_{s}=V_{c s}^{*} V_{u s}, \quad \lambda_{b}=V_{c b}^{*} V_{u b},
$$

and CKM unitarity $\lambda_{d}+\lambda_{s}+\lambda_{b}=0$ is invoked to eliminate one combination of CKM elements. A common choice for the decomposition of an SCS decay amplitude is

$$
\mathscr{A}^{\mathrm{SCS}} \equiv \lambda_{s d} A_{s d}-\frac{\lambda_{b}}{2} A_{b}
$$

with

$$
\lambda_{s d}=\frac{\lambda_{s}-\lambda_{d}}{2} \quad \text { and } \quad-\frac{\lambda_{b}}{2}=\frac{\lambda_{s}+\lambda_{d}}{2} .
$$

In view of $\left|\lambda_{b}\right| /\left|\lambda_{s d}\right| \sim 10^{-3}$ only $A_{s d}$ is relevant for branching ratios. Within the SM a nonvanishing direct CP asymmetry involves the interference of $\lambda_{b} A_{b}$ with $\lambda_{s d} A_{s d}$. Neglecting quadratic (and higher) terms in $\lambda_{b} / \lambda_{s d}$ the direct CP asymmetry reads

$$
a_{C P}^{\mathrm{dir}}=\operatorname{Im} \frac{\lambda_{b}}{\lambda_{s d}} \operatorname{Im} \frac{A_{b}}{A_{s d}}
$$


Using $\mathscr{A}^{\mathrm{SCS}} \simeq \lambda_{s d} A_{s d}$ and the standard CKM phase convention with (essentially) $\lambda_{s d}>0$ Eq. (1.4) becomes

$$
a_{C P}^{\mathrm{dir}}=\frac{\operatorname{Im} \lambda_{b}}{\left|\mathscr{A}^{\mathrm{SCS}}\right|} \operatorname{Im} \frac{A_{b}}{A_{s d}}\left|A_{s d}\right|
$$

Recalling that $\left|\mathscr{A}^{\mathrm{SCS}}\right|$ is determined by the well-measured branching ratio of the considered decay we realize from Eq. (1.5) that two non-trivial imputs are needed to predict $a_{C P}^{\mathrm{dir}}:\left|A_{b}\right|$ and the relative phase between $A_{b}$ and $A_{s d}$. The latter is a CP-conserving (strong) phase; the CP-violating (weak) phase is $\arg \left(\lambda_{b} / \lambda_{s d}\right)$.

It is not possible to calculate $\left|A_{b}\right|$ and $\arg \left(A_{b} / A_{s d}\right)$ from first principles. The theoretical method of choice in charm physics is the approximate $\mathrm{SU}(3)_{F}$ symmetry of QCD, which permits to correlate the amplitudes of different decays with each other. $\mathrm{SU}(3)_{F}$ symmetry refers to unitary rotations among up, down, and strange fields and would be exact in the limit $m_{u}=m_{d}=m_{s}$ of equal light-quark masses. The parameter determining the size of $\mathrm{SU}(3)_{F}$ breaking is $\left(m_{s}-m_{d}\right) / \Lambda_{\mathrm{QCD}}=$ $\mathscr{O}(30 \%)$, where $\Lambda_{\mathrm{QCD}}$ is the fundamental scale of QCD. The actual accuracy of SU(3) Fymmetry varies among different observables and it is desirable to include first-order (linear) $\mathrm{SU}(3)_{F}$ breaking.

\section{Sum rules of $\mathrm{CP}$ asymmetries}

$\mathrm{SU}(3)_{F}$ analyses can be done in two ways: First, one may express the physical decay amplitudes in terms of group-theoretical objects, the reduced amplitudes, which correspond to different representations of $\mathrm{SU}(3)$. It is possible to include first-order $\mathrm{SU}(3)_{F}$ breaking at the expense of having to deal with more reduced amplitudes (see e.g. $[1,7,16]$ ). Second, one can instead express the $D$ decay amplitudes in terms of topological amplitudes, which are classified by the flavour flow [18-20]. Also this method allows for the inclusion of first-order SU(3) $F$ breaking [21,22]. The topological amplitudes are ilustrated in Fig. 1. At this stage both methods are mathematically equivalent [22]. However, the topogical-amplitude method has the advantage that it can be combined with large- $N_{c}$ counting $[23,24]$ to sharpen the theoretical predictions. Here $N_{c}=3$ is the number of colors and to leading order in the $1 / N_{c}$ expansion the $T$ and $A$ amplitudes can be expressed in term of form factors and decay constants.

Armed with this formalism we can study branching fractions and CP asymmetries of $D \rightarrow$ $P P^{\prime}$ decays. These observables play very different roles in charm physics: Branching ratios of hadronic charm decays are "dull" tree-level quantities dominated by a single CKM amplitude and are therefore insensitive to new physics. They are useful to test the calculational framework and experimentally determine $\left|A_{s d}\right|$, which is one of the ingredients to predict $\mathrm{CP}$ asymmetries. On the contrary, CP asymmetries of hadronic charm decays are suppressed by $\operatorname{Im} \frac{\lambda_{b}}{\lambda_{s d}}=-6 \cdot 10^{-4}$ in the Standard Model and therefore probe new physics in flavour transitions of up-type quarks. When exploiting the experimental information on $16 D \rightarrow P P^{\prime}$ branching fractions and the $D^{0} \rightarrow$ $K^{ \pm} \pi^{\mp}$ strong-phase difference to predict CP asymmetries, one faces a fundamental problem: CP asymmetries involve topological amplitudes (equivalently, reduced $\mathrm{SU}(3)_{F}$ amplitudes) which do not enter branching ratios and are therefore unconstrained by any global fit to the corresponding data. The most prominent example is the penguin amplitude: Denoting the penguin with internal 


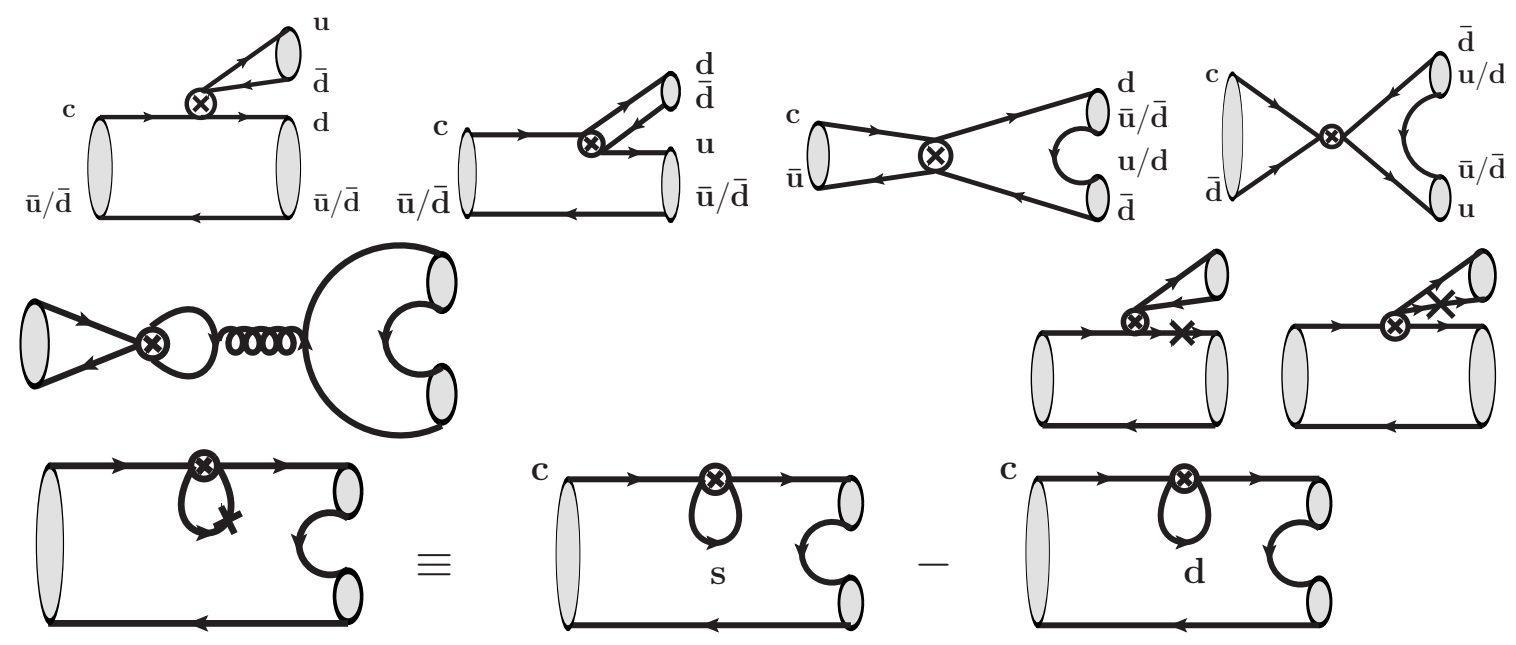

Figure 1: First row: SU(3) limit topological amplitudes "tree" (T), "color-suppressed tree" (C), "exchange" (E), "annihilation" (A). Second row: penguin annhilation (PA) diagram and examples of topological amplitudes describing SU(3) $)_{F}$-breaking effects (with the cross distinguishing an $s$ from a $d$ or $u$ line); the depicted

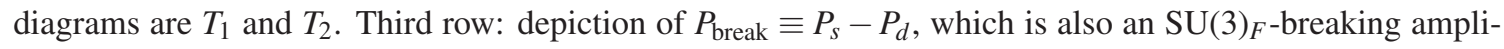
tude.

quark $q$ by $P_{q}$, the branching fractions only constrain the (SU(3) $F$-breaking) combination $P_{\text {break }} \equiv$ $P_{s}-P_{d}$. CP asymmetries, however, involve not only $P_{\text {break }}$ but also $P \equiv P_{s}+P_{d}-2 P_{b}$.

Since we cannot predict individual $\mathrm{CP}$ asymmetries, we may next try to predict relations (sum rules) between different $\mathrm{CP}$ asymmetries. In the limit of exact $\mathrm{SU}(3)_{F}$ symmetry there are two sum rules among two direct $\mathrm{CP}$ asymmetries each [13]:

$$
\begin{aligned}
& a_{C P}^{\mathrm{dir}}\left(D^{0} \rightarrow K^{+} K^{-}\right)+a_{C P}^{\mathrm{dir}}\left(D^{0} \rightarrow \pi^{+} \pi^{-}\right)=0, \\
& a_{C P}^{\mathrm{dir}}\left(D^{+} \rightarrow \bar{K}^{0} K^{+}\right)+a_{C P}^{\mathrm{dir}}\left(D_{s}^{+} \rightarrow K^{0} \pi^{+}\right)=0 .
\end{aligned}
$$

In Ref. [17] it has been shown that there are no sum rules among CP asymmetries which hold to first order in $\mathrm{SU}(3)_{F}$ breaking. Can we improve on Eqs. (2.1) and (2.2) anyway? To this end consider, for example,

$$
A_{s d}\left(D^{0} \rightarrow \pi^{+} \pi^{-}\right)=-T-E+P_{\text {break }}, \quad A_{b}\left(D^{0} \rightarrow \pi^{+} \pi^{-}\right)=T+E+P+P A,
$$

which entails $A_{b}\left(D^{0} \rightarrow \pi^{+} \pi^{-}\right)=-A_{s d}\left(D^{0} \rightarrow \pi^{+} \pi^{-}\right)+P_{\text {break }}+P+P A$. (PA is defined analogously to $P$.) Then Eq. (1.4) reads

$$
a_{C P}^{\mathrm{dir}}\left(D^{0} \rightarrow \pi^{+} \pi^{-}\right)=\operatorname{Im} \frac{\lambda_{b}}{\lambda_{s d}} \operatorname{Im} \frac{P_{\text {break }}+P+P A}{A_{s d}\left(D^{0} \rightarrow \pi^{+} \pi^{-}\right)} .
$$

In the $\mathrm{SU}(3)_{F}$ limit the corresponding expression for $a_{C P}^{\mathrm{dir}}\left(D^{0} \rightarrow K^{+} K^{-}\right)$is indeed equal in magnitude and opposite in sign. One can next use the global branching-ratio analysis of Ref. [22] to determine all ingredients of $a_{C P}^{\mathrm{dir}}\left(D^{0} \rightarrow \pi^{+} \pi^{-}\right)$and $a_{C P}^{\mathrm{dir}}\left(D^{0} \rightarrow K^{+} K^{-}\right)$including first-order $\mathrm{SU}(3)_{F}$ breaking, except for $P+P A$ which is unconstrained. I.e. we are left with two quantities depending 


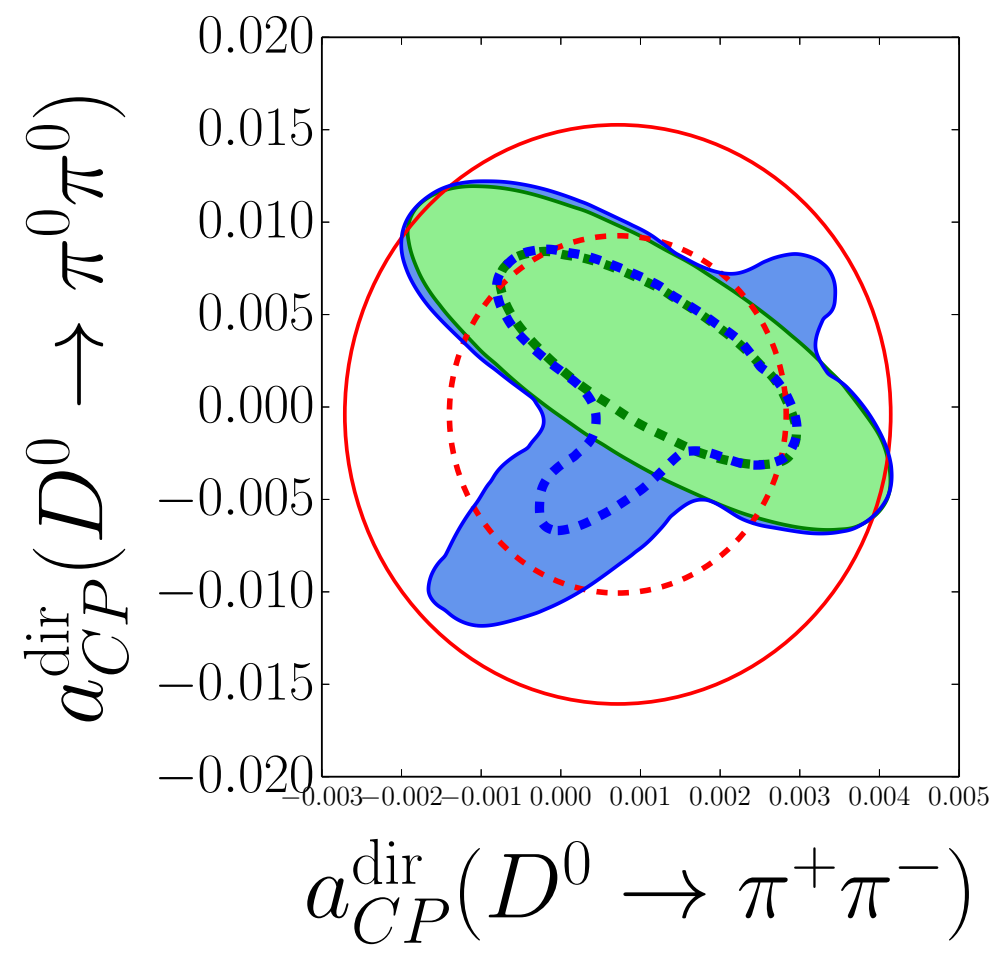

Figure 2: The solid (dashed) lines delimit the 95\% (68\%) CL region. Red: direct measurements. Blue: prediction of sum rule with present data. Green: sum rule if branching ratios were improved by a factor of $\sqrt{50}$ compared to today. Plot from Ref. [25].

on two unknowns, which are real and imaginary part of $P+P A$. In order to make a prediction we therefore need a third a quantity, $a_{C P}^{\mathrm{dir}}\left(D^{0} \rightarrow \pi^{0} \pi^{0}\right)$. By eliminating $P+P A$ we find the desired sum rule. The global fit à la Ref. [22] determines all topological amplitudes entering $A_{s d}$ for the three decay modes (which are $T, E, C, T_{1,2}, E_{1,2}$, and $P_{\text {break }}$ ), so that the troublesome $\mathrm{SU}(3)_{F}$-breaking terms causing $B\left(D^{0} \rightarrow K^{+} K^{-}\right) \neq B\left(D^{0} \rightarrow \pi^{+} \pi^{-}\right)$are taken care of. However, $P+P A$ cannot be treated beyond the $\mathrm{SU}(3)_{F}$ limit. Fig. 2 shows the impact of the sum rule. Similarly, we can improve Eq. (2.2) to a sum rule involving also the third decay mode $D_{s}^{+} \rightarrow K^{+} \pi^{0}$. With current data the sum rule has much larger errors than the one for $D^{0} \rightarrow K^{+} K^{-}, \pi^{+} \pi^{-}, \pi^{0} \pi^{0}$.

\section{CP asymmetry in $D^{0} \rightarrow K_{S} K_{S}$}

$D^{0} \rightarrow K_{S} K_{S}$ has the special feature that $A_{s d}$ vanishes in the $\mathrm{SU}(3)_{F}$ limit. The smallness of $\left|A_{s d}\right|$ enhances $A_{b} / A_{s d}$, so that $a_{C P}^{\mathrm{dir}}$ in Eq. (1.4) is expected to be larger than in other decays. Concerning statistical errors this observation does not help, because the gain in statistical significance is cancelled by the smaller number of events, which scale with the branching ratio proportional to $\left|A_{s d}\right|^{2}$. In our case we have $B\left(D^{0} \rightarrow K_{S} K_{S}\right)=(1.7 \pm 0.4) \cdot 10^{-4}$. Still, a larger CP asymmetry may help to fight systematic errors. Another special feature of the considered decay mode is way more interesting: The numerator $A_{b}$ in Eq. (1.4) receives contributions from the exchange diagram $E$, so that the $\mathrm{CP}$ asymmetry persists even if the loop-induced amplitudes $P$ and $P A$ (which induce the $\mathrm{CP}$ asymmetries in essentially all other decay modes) turn out to be tiny. Moreover, the global fit 
of Ref. [22] points to a large $E$ and definitely excludes $E=0$. The sensitivity to $E$ stems from the feature that in $D^{0} \rightarrow K_{S} K_{S}$ the transitions $c \bar{u} \rightarrow s \bar{s}$ and $c \bar{u} \rightarrow d \bar{d}$ can interfere, because both $s \bar{s}$ and $d \bar{d}$ can hadronize into a $K_{S} K_{S}$ pair. In Ref. [26] we find

$$
-1.1 \cdot 10^{-2} \leq a_{C P}^{\mathrm{dir}} \leq 1.1 \cdot 10^{-2} .
$$

This number assumes that the $\mathrm{CP}$ asymmetry related to Kaon mixing is properly subtracted. Unfortunately, the global fit to all $D \rightarrow P P^{\prime}$ data presently does not rule out that $\left|a_{C P}^{\mathrm{dir}}\right|$ is much smaller than $1.1 \cdot 10^{-2}$. One source of uncertainty is the strong phase $\arg \left(A_{b} / A_{s d}\right)$ which is currently unconstrained. To eliminate this source of uncertainty, one must also measure the mixing-induced $\mathrm{CP}$ asymmetry [26], with a time-dependent measurement or through CP-tagged decays. Currently experiments determine

$$
A_{C P}=a_{C P}^{\mathrm{dir}}-A_{\Gamma} \frac{\langle t\rangle}{\tau}
$$

where $\langle t\rangle$ is the average decay time and $\tau$ is the $D^{0}$ lifetime. $A_{\Gamma}$ involves the mixing-induced $\mathrm{CP}$ asymmetry and is small, because $D^{0}$ mesons oscillate very slowly. The experimental results are [27-29]

$$
\begin{aligned}
A_{C P}^{\mathrm{CLEO} 2001} & =-0.23 \pm 0.19, \quad A_{C P}^{\mathrm{LHCb} 2015}=-0.029 \pm 0.052 \pm 0.022 \\
A_{C P}^{\text {Belle } 2016} & =-0.0002 \pm 0.0153 \pm 0.0017 .
\end{aligned}
$$

\section{Summary}

$\mathrm{CP}$ asymmetries in $D$ decays involve topological amplitudes which are not constrained by fits to branching ratio data. These can be eliminated by forming judicious combinations (sum rules) of several $\mathrm{CP}$ asymmetries. Within the limits of expected $\mathrm{SU}(3)_{F}$ breaking in penguin (and penguin annihilation) amplitudes these sum rules probe new physics. Within the Standard Model the direct $\mathrm{CP}$ asymmetry in $D^{0} \rightarrow K_{S} K_{S}$ can be as large as $1.1 \%$. $a_{C P}^{\mathrm{dir}}\left(D^{0} \rightarrow K_{S} K_{S}\right)$ is dominated by the exchange diagram, which involves no loop suppression. We advocate $D^{0} \rightarrow K_{S} K_{S}$ as a potential discovery channel for charm $\mathrm{CP}$ violation.

\section{Acknowledgements}

I thank the organisers for inviting me to this talk. The presented work is supported by BMBF under contract 05H12VKF. Parts of the computations were performed on the NEMO cluster of Baden-Württemberg (framework program bwHPC).

\section{References}

[1] G. Hiller, M. Jung and S. Schacht, Phys. Rev. D 87 (2013) no.1, 014024 doi:10.1103/PhysRevD.87.014024 [arXiv:1211.3734 [hep-ph]].

[2] E. Franco, S. Mishima and L. Silvestrini, JHEP 1205 (2012) 140 doi:10.1007/JHEP05(2012)140 [arXiv:1203.3131 [hep-ph]]. 
[3] H. n. Li, C. D. Lu and F. S. Yu, Phys. Rev. D 86 (2012) 036012 doi:10.1103/PhysRevD.86.036012 [arXiv:1203.3120 [hep-ph]].

[4] J. Brod, Y. Grossman, A. L. Kagan and J. Zupan, JHEP 1210 (2012) 161 doi:10.1007/JHEP10(2012)161 [arXiv:1203.6659 [hep-ph]].

[5] T. Feldmann, S. Nandi and A. Soni, JHEP 1206 (2012) 007 doi:10.1007/JHEP06(2012)007 [arXiv:1202.3795 [hep-ph]].

[6] H. Y. Cheng and C. W. Chiang, Phys. Rev. D 85 (2012) 034036 Erratum: [Phys. Rev. D 85 (2012) 079903] doi:10.1103/PhysRevD.85.079903, 10.1103/PhysRevD.85.034036 [arXiv:1201.0785 [hep-ph]].

[7] D. Pirtskhalava and P. Uttayarat, Phys. Lett. B 712 (2012) 81 doi:10.1016/j.physletb.2012.04.039 [arXiv:1112.5451 [hep-ph]].

[8] J. Brod, A. L. Kagan and J. Zupan, Phys. Rev. D 86 (2012) 014023 doi:10.1103/PhysRevD.86.014023 [arXiv:1111.5000 [hep-ph]].

[9] I. I. Bigi, A. Paul and S. Recksiegel, JHEP 1106 (2011) 089 doi:10.1007/JHEP06(2011)089 [arXiv:1103.5785 [hep-ph]].

[10] M. Bobrowski, A. Lenz, J. Riedl and J. Rohrwild, JHEP 1003 (2010) 009 doi:10.1007/JHEP03(2010)009 [arXiv:1002.4794 [hep-ph]].

[11] A. A. Petrov, PoS BEAUTY 2009 (2009) 024 [arXiv:1003.0906 [hep-ph]].

[12] M. Artuso, B. Meadows and A. A. Petrov, Ann. Rev. Nucl. Part. Sci. 58 (2008) 249 doi:10.1146/annurev.nucl.58.110707.171131 [arXiv:0802.2934 [hep-ph]].

[13] Y. Grossman, A. L. Kagan and Y. Nir, Phys. Rev. D 75 (2007) 036008 doi:10.1103/PhysRevD.75.036008 [hep-ph/0609178].

[14] F. Buccella, M. Lusignoli, G. Miele, A. Pugliese and P. Santorelli, Phys. Rev. D 51 (1995) 3478 doi:10.1103/PhysRevD.51.3478 [hep-ph/9411286].

[15] M. Golden and B. Grinstein, Phys. Lett. B 222 (1989) 501. doi:10.1016/0370-2693(89)90353-5

[16] Y. Grossman and D. J. Robinson, JHEP 1304 (2013) 067 doi:10.1007/JHEP04(2013)067 [arXiv:1211.3361 [hep-ph]].

[17] Y. Grossman, Z. Ligeti and D. J. Robinson, JHEP 1401 (2014) 066 doi:10.1007/JHEP01(2014)066 [arXiv:1308.4143 [hep-ph]].

[18] L. L. C. Wang, Flavor Mixing and Charm Decay, talk at the Conference on Theoretical Particle Physics, 5-14 January 1980, Guangzhou (Canton), China, p. 1218, report BNL-27615, C80-01-05-20.

[19] L. L. Chau, Phys. Rept. 95 (1983) 1. doi:10.1016/0370-1573(83)90043-1

[20] D. Zeppenfeld, Z. Phys. C 8 (1981) 77. doi:10.1007/BF01429835

[21] M. Gronau, O. F. Hernandez, D. London and J. L. Rosner, Phys. Rev. D 52 (1995) 6356 doi:10.1103/PhysRevD.52.6356 [hep-ph/9504326].

[22] S. Müller, U. Nierste and S. Schacht, Phys. Rev. D 92 (2015) no.1, 014004 doi:10.1103/PhysRevD.92.014004 [arXiv:1503.06759 [hep-ph]].

[23] A. J. Buras, J. M. Gerard and R. Ruckl, Nucl. Phys. B 268 (1986) 16. doi:10.1016/0550-3213(86)90200-2 
[24] A. J. Buras and L. Silvestrini, Nucl. Phys. B 569 (2000) 3 doi:10.1016/S0550-3213(99)00712-9 [hep-ph/9812392].

[25] S. Müller, U. Nierste and S. Schacht, Phys. Rev. Lett. 115 (2015) no.25, 251802 doi:10.1103/PhysRevLett.115.251802 [arXiv:1506.04121 [hep-ph]].

[26] U. Nierste and S. Schacht, Phys. Rev. D 92 (2015) no.5, 054036 doi:10.1103/PhysRevD.92.054036 [arXiv:1508.00074 [hep-ph]].

[27] G. Bonvicini et al. [CLEO Collaboration], Phys. Rev. D 63 (2001) 071101 doi:10.1103/PhysRevD.63.071101 [hep-ex/0012054].

[28] R. Aaij et al. [LHCb Collaboration], JHEP 1510 (2015) 055 doi:10.1007/JHEP10(2015)055 [arXiv:1508.06087 [hep-ex]].

[29] A. Abdesselam et al., Measurement of CP asymmetry in the $D^{0} \rightarrow K_{S}^{0} K_{S}^{0}$ decay at Belle, talk at 38th International Conference on High Energy Physics (ICHEP2016), 3-10 August 2016, Chicago, USA, arXiv:1609.06393 [hep-ex]. 